\title{
A FAMILY OF SPREAD-SEQUENCES FOR CDMA SYSTEM IN A MULTIPATH FADING CHANNEL
}

\author{
A.Nallanathan, S.C.Chan, T.S.Ng \\ Department of Electrical and Electronic Engineering \\ University of Hong Kong, \\ Pokfulam Road, \\ Hong Kong. \\ e-mail: analla@eee.hku.hk, scchan@eee.hku.hk, tsng@eee.hku.hk
}

\begin{abstract}
A new class of orthogonal code-division multiple access (CDMA) system is developed. The key characteristic of the system is that the data symbols are spreaded by a spread-sequence that is longer than the period of the symbol and hence overlapped with the neighboring symbols. Using this approach, temporal diversity is incorporated with other diversities. Due to the temporal diversity, the proposed CDMA system performs well in fading environment. In this paper, a method for designing such a spread-sequence using filter bank theory is presented. The length of the spreadsequence could be varied according to the requirement. Simulation results show that the proposed spreadsequence based system yields lower BER than the conventional gold codes based DS/CDMA system.
\end{abstract}

\section{INTRODUCTION}

There has been increasing interest recently in using direct sequence spread spectrum (DS-SS) code division multiple access (CDMA) for commercial applications. DS/CDMA is proposed for cellular, microcellular, indoor and satellite communications [6]. CDMA is also a candidate for high data rate applications, such as wireless local area network (LAN) and videophones. Very often the SS modulation is used to mitigate the different problems encountered in different communications media. Therefore it is desirable to have a flexible and efficient design method for this signal suit various communication channel conditions. In this paper, a class of orthogonal CDMA system using longer and overlapping spread-sequences is proposed. Since the sequences are lapped, a greater temporal diversity is achieved and the bandwidth of the system is maintained as the conventional DS/CDMA system. In order to design spread-sequences with binary values, the length of the spread-sequence proposed in [8] increases exponentially with the number of users. This causes large system delay in transmission. To avoid the large delay, we propose a new structure for constructing spread-sequences with flexible length. This greatly widens the selection of spread-sequences to suit different system delay requirement. On the other hand, the sequence will assume integer values instead of binary values. The paper is organized as follows: Section II is devoted to the system model of the new class of CDMA system. The channel model is described in Section III. In Section IV, methods for constructing spread-sequences will be discussed and a new class of spread-sequence will be introduced. Simulation results are given in section V. Finally, the conclusion is given in section VI.

\section{SYSTEM MODEL}

The system model of the proposed orthogonal CDMA system is shown in Fig 1. The input data symbols, $b_{m}[n]$, are assumed to be binary antipodal where $m$ stands for the $m$ th user. Each data symbols, $b_{m}[n]$, is modulated onto a unique spread-sequence $h_{m}[n]$ of length $p M(p>1)$ where $p$ is an integer and $M$ is the chip length of conventional system. The modulated signal is transmitted within the total available bandwidth. We can view the modulation process as consisting of two stages. As depicted in Fig. 1, these stages correspond to upsampling by a factor of $M$ followed by linear time-invariant filtering with the spread-sequence. The key motivation for using longer sequence is that they can lead to a greater temporal diversity.

\section{CHANNEL MODEL}

Several multipath models have been proposed in the literature, varying from the very comprehensive model to the simple tapped delay line model [6]. It has been shown that the performance of the DS/CDMA system is robust with respect to channel models. In this paper, the multipath fading channel is modeled by a continuum and a fixed number of paths. Utilizing power control among the CDMA users, the channel is assumed to be statistically identical for all users. The lowpass equivalent impulse response of the pass band fading channel is given by [6], 


$$
h(t)=\sum_{l=1}^{L_{p}} \beta_{l} \delta\left(t-\tau_{l}\right) e^{y_{l}}
$$

where, $L_{p}$ is the number of paths, $f_{l}, \tau_{l}$ and $\gamma_{l}$ are respectively the path gain, delay and phase of the $l$ th path. The channel auto-covariance function is easily found to be,

$$
\mu(t)=\sum_{l=1}^{L_{p}} \sigma_{l}^{2} \delta\left(t-\tau_{l}\right)
$$

The unit energy constraint on the fading process covariance function implies that

$$
\sum_{i=1}^{L_{p}} \sigma_{i}^{2}=1
$$

where the variance, $\sigma_{l}^{2}$, for uniform and exponential multipath power profiles are given respectively by,

$$
\sigma_{l}^{2}=\frac{1}{L_{p}} \quad \text { and } \quad \sigma_{l}^{2}=\sigma_{1}^{2} e^{-\frac{l-1}{L^{\prime}}}
$$

The parameter $L^{\prime}$ decides the rate of power decay on the successive paths. Using (3.2) and (3.3) for the exponential profile and arbitrarily taking $L^{\prime}=L_{p}$, one gets,

$$
\sigma_{1}^{2}=\frac{1-e^{-1 / L_{p}}}{1-e^{-1}}
$$

\section{SPREAD-SEQUENCES}

In multiple access systems, the coded symbol stream of the $m$ th user, $b_{m}(n)$, is modulated onto a unique spread-sequence $h_{m}(n)$ to produce $y_{m}(n)$ which is transmitted within the available system bandwidth. In the conventional multiuser system the maximum number of users is equal to the chip length $M$. However we can use the lapped orthogonal spreadsequence with the length of integer multiple of $M$. A natural requirement of such systems is that, in the absence of fading and with perfect synchronization among users, there will be no intersymbol interference either within a user's stream or among users. This is equivalent to requiring that the sequence sets satisfy certain orthogonality conditions. A reasonable solution is to ensure that the sequence together with all their translates by integer multiples of $M$ constitute an orthonormal basis. If the spread-sequences are written as a vector sequence,

$$
\mathbf{h}[n]=\left[h_{1}[n], h_{2}[n] \cdots \cdots \cdots \cdots h_{M}[n]\right]^{\mathrm{P}} .
$$

The orthogonality condition can be expressed as,

$$
\sum_{k} \mathbf{h}[k-n M] \mathbf{h}^{\mathrm{T}}[k-l M]=\delta[n-l) \mathbf{I}_{M}
$$

where $\mathbf{I}_{M}$ denotes the identity matrix of appropriate size and $\delta[n]$ denotes the unit impulse. The modulated signal can then be written as follows,

$$
y_{m}[n]=\sum_{k} x_{m}[k] h_{m}[n-k M] .
$$

The design of such sequence sets is related to the theory of perfect reconstruction filter bank. In what follows, it will be shown the orthogonality condition in (4.2) can also be interpreted as the PR condition of a multirate filter bank made up of $h_{i}[n]$.

Let's consider the z-transform of equation (4.1) as follows,

$$
\begin{aligned}
\mathbf{H}(z) & =\sum_{n} \mathbf{h}[n] z^{-n} \\
& =\left[\begin{array}{lll}
H_{1}(z) & H_{2}(z) \cdots \cdots H_{M}(z)
\end{array}\right]^{T},
\end{aligned}
$$

where $H_{i}(z)=\sum_{n} h_{i}[n] z^{-n}$.

Using type-1 polyphase decomposition [1], $\mathbf{H}(z)$ can be written in the following form,

$$
\mathbf{H}(z)=\mathbf{Q}\left(z^{M}\right) \mathbf{e}(z),
$$

where $\mathbf{Q}(z)$ is the polyphase matrix and $\mathrm{e}(z)$ is the $z$ transform of the delay chain of order $M$, i.e,

$$
\mathrm{e}(z)=\left[\begin{array}{llll}
1 & z^{-1} & z^{-2} \cdots \cdots \cdot z^{-(M-1)}
\end{array}\right]^{T} .
$$

For orthonormal sequence sets, the associated polyphase matrix satisfies the following property,

$$
\mathbf{Q}(z) \mathbf{Q}(z)^{* T}=\mathbf{I}_{\mathbf{M}} .
$$

where * and $T$ denote complex conjugation and matrix transposition respectively. Equation (4.7) is equivalent to the PR condition of the filter bank consisting of $h_{i}[n]$ and matrices which satisfy $(4.7)$ are referred to as "paraunitary".

The Auto- and cross-correlation characteristics of the spread-sequences are two very important properties in a 
CDMA system. Indeed, the auto correlation characteristics generally affect, for example, the ability of the receiver to synchronize the transmission, while the cross-correlation characteristics generally affect the degree and nature of co-channel interference. Ideally, one would like the auto correlation of each sequence $h_{k}[n]$ to satisfy,

$$
h_{k}[n] * h_{k}[n] \approx \delta[n],
$$

and the cross correlation between distinct sequences $h_{k}[n]$ and $h_{l}[n]$ to satisfy,

$$
h_{k}[n] * h_{l}[-n] \approx 0 \quad k \neq l
$$

However (4.8) and (4.9) are conflicting objectives for traditional sequence sets. This is also true for spreadsequence sets. In fact, it was shown in [8] that good auto-correlation characteristics can only be obtained at the expense of cross-correlation characteristics, and vice versa. At one extreme, the trivial signature set corresponding to TDMA systems has perfect autocorrelation characteristics, but the worst possible crosscorrelation characteristics. At the other extreme, the sequence sets corresponding to ideal FDMA system has perfect cross-correlation characteristics but poor autocorrelation characteristics. In practical CDMA system, a compromise between these extremes is generally sought.

The auto-correlation and the cross-correlation characteristics constitute only one of the important issue in the design of good spread-sequence sets. It is also important that the sequence sets be effective in mitigating the effects of fading by spreading each symbol bits of a user over a range of time samples. The dispersion factor $D_{h}$ which measures a sequence set's spreading capability is defined as [8],

$$
\frac{1}{D_{h}}=\frac{1}{M} \sum_{m} \frac{1}{D_{h_{m}}},
$$

where $D_{h_{m}}$ represents the dispersion in the sequence $h_{m}[n]$, i.e,

$$
D_{h_{m}}=\left(\sum_{m} h_{m}^{4}[n]\right)^{-1} .
$$

\section{a. A Class of Spread-Sequence Set}

In [8], Wornell proposed a family of orthogonal sequence set that is optimal in the sense of being maximally spread. The sequence set was obtained from a cascade structure using Hadamard matrix which has a very low implementation complexity. More precisely, the polyphase matrix is obtained by repeated cascading of lower order polyphase matrix as follows,

$$
\mathbf{Q}^{(i)}(z)=\equiv \Lambda\left(z^{M^{(i-1)}}\right) \mathbf{Q}^{(i-1)}(z), i=1,2 \ldots \ldots
$$

where $\Lambda(z)$ is the diagonal delay matrix whose diagonal is constructed from the elements of $\mathbf{e}(z)$, i.e,

$$
\Lambda(z)=\operatorname{diag}(\mathbf{e}(z))
$$

From (4.5) and (4.12), the spread-sequence sets are given by,

$$
\mathbf{H}^{(i)}(z)=\Xi \Lambda\left(z^{M^{i}}\right) \mathbf{H}^{(i-1)}(z) .
$$

$\Xi$ is the $(M \times M)$ Hadamard matrix and $Q^{(0)}(z)$ is chosen to be the Hadamard matrix. i.e., $Q^{(0)}(z)=\Xi_{M}$.

\section{b. Proposed Spread-Sequence Set}

From (4.7), we notice that the design of orthonormal sequence set is equivalent to the determination of paraunitary matrix, $Q(z)$, that satisfies the $P R$ condition. In [8], the sequence set is constructed from (4.12), which yield sequence set that assumes binary values of +1 or -1 . However the length of the constructed sequence set increases exponentially with $M$. In fact, the length of the signature set is $M^{k}$, $k=2,3 \ldots .$. . To avoid the long system delay, spread sequence set can be derived from the general solution of (4.14). The length of such signature set is more flexible. i.e, $M, 2 M, \ldots \ldots$. On the other hand, the values of the signature set will no longer be +1 or -1 .

The complete factorization of paraunitary matrices has been studied previously in [1],[10]. Our proposed construction follows a related factorization of the paraunitary matrix derived in [10]. Spread-sequence set with length $L=p M$ is constructed from the following cascade structure,

$$
\mathbf{Q}(z)=\mathbf{K}_{p-1}(z) \mathbf{K}_{p-2}(z) \ldots \ldots \mathbf{K}_{1}(z) \Xi_{M},
$$

where

$$
\begin{aligned}
& \mathbf{K}_{i}(z)=\mathbf{P}_{i} \Phi_{i} \mathbf{W} \Omega(z) \mathbf{W}, \\
& \mathbf{W}=\frac{1}{\sqrt{2}}\left[\begin{array}{cc}
\mathbf{I}_{N / 2} & \mathbf{I}_{N / 2} \\
\mathbf{I}_{N / 2} & -\mathbf{I}_{N / 2}
\end{array}\right],
\end{aligned}
$$




$$
\begin{array}{r}
\Omega(z)=\left[\begin{array}{ll}
\mathbf{I}_{N / 2} & \mathbf{O}_{N / 2} \\
\mathbf{O}_{N / 2} & z^{-1} \mathbf{I}_{N / 2}
\end{array}\right], \\
\boldsymbol{\Phi}_{i}=\left[\begin{array}{cc}
\mathbf{U}_{i} & \mathbf{O}_{N / 2} \\
\mathbf{O}_{N / 2} & \mathbf{V}_{i}
\end{array}\right] .
\end{array}
$$

$\mathbf{U}_{i}, \quad \mathbf{V}_{i}$ can be any $(M / 2) \times(M / 2)$ orthogonal matrices. For simlplicity of implementation, they are taken to be the Hadamard matrix $\Xi_{M / 2} . \mathbf{P}_{i}$ is a random permutation matrix which is used to randomize the spread- sequences.

\section{SIMULATION RESULTS}

In this section, the performance of the conventional DS/CDMA and the proposed Spread-Sequence based CDMA system is compared. Gold codes with length 32 are used for the simulation of the conventional DS/CDMA system. The proposed sequence set with different overlapping factors is used for the spread sequence based CDMA system and they are obtained using equation (4.15). Fig.2a and Fig.2b show the spectrum of two proposed spread-sequences for $M=32$ with $p=4$. It can be seen that the proposed spread sequence set is neither frequency or time localized.

The channel is assumed to be Rayleigh fading with exponential 'power delay profile' ( 2 rays). To evaluate the performance of the proposed system, BER performance against $E_{b} / N_{o}$ is simulated and compared with the conventional DS/CDMA system. Throughout the simulation, the RAKE receiver (2-finger) is used. Fig.3 shows the BER performance of the two systems against $E_{b} / N_{o}$ when the number of users is 20 for uplink (asynchronous system). Fig.4 shows the BER performance of the two systems against $E_{b} / N_{o}$ when the number of users is 20 for down-link (synchronous system). The improvement of the proposed spreadsequence modulation can partially be explained by the incorporation of time diversity in addition to path diversity. In Practice, RAKE receiver implementation may be expensive and match filters can be employed at the expense of BER performance.

\section{CONCLUSION}

A new class of spread-sequence based orthogonal code-division multiple access (CDMA) system is developed. A method for designing such a spreadsequence set is proposed using filter bank theory. It is derived from the factorization of the paraunitary matrices in [10]. As compared with the sequence set proposed in [8], more freedom in selecting the length of the spread-sequences can be achieved but the resulting spread-sequences become integer valued. Simulation results show that the BER performance of the proposed Spread-Sequence based CDMA system is better than the conventional DS/CDMA system. Such improvement also increases with the overlapping length of the spread signature set.

\section{ACKNOWLEDGEMENT}

This work is supported by the Hong Kong Research Grants Council and the CRCG of the University of Hong Kong. The first author would like to thank Dr.E.H.Li, Department of Electrical and Electronic Engineering, University of Hong Hong, for providing financial support to attend the conference.

\section{REFERENCES}

(1) Vaidyanathan, P.P., "Multirate systems and filter banks". PTR Printice Hall, 1993.

(2) Nallanathan, A., Chan,S.C. and Ng,T.S., "Generalized Lapped Transform (GLT) based highspeed transmission for wireless communications", Proc. IEEE VTC'98, pp 1409-1413, Ottawa, May 1998.

(3) Nallanathan,A., Chan,S.C. and Ng,T.S., "Generalized lapped Transform (GLT) based codes for multi-user communications" in Proc. Fifth symposium on Communications and Vehicular Technology, Netherland, Oct 1997.

(4) Chan.S.C., Nallanathan,A., and Ng,T.S., " A Class of M-channel linear-phase biorthogonal filter banks and their applications to subband coding" to appear in IEEE Trans. on Signal Processing, February 1999.

(5) Chan.S.C., "The Generalized lapped Transform (GLT) for Subband coding Applications, "Proc.IEEE ICASSP'95, pp 1508-1511, May 1995.

(6) Prasad,R.," CDMA for Wireless Personal communications. Artech House". 1996.

(7) Malvar,H.S., “ Signal Processing with lapped Transforms", Artech House, 1992.

(8) Wornell,G.W., " Spread-Signature CDMA: Efficient Multiuser Communication in the Presence of Fading", IEEE Trans on Information Theory, Vol 41, No.5, September 1995.

(9) Wornell, G.W., " Spread-Response precoding for Communication over fading Channels ", IEEE Trans on Information Theory, Vol. 42, No.2, March 1996.

(10) Soman,A.K., Vaidyanathan,P.P., and Nguyen,T.Q., " Linear Phase Paraunitary Filter Banks: Theory, Factorizations and Designs" IEEE Trans on Signal Processing, Vol 41, No12, December 1993. 


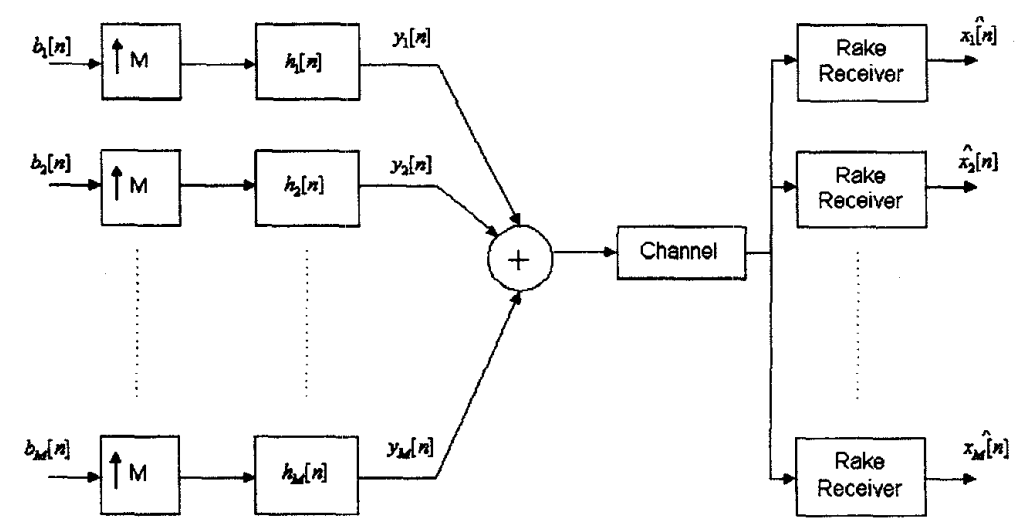

Fig.1

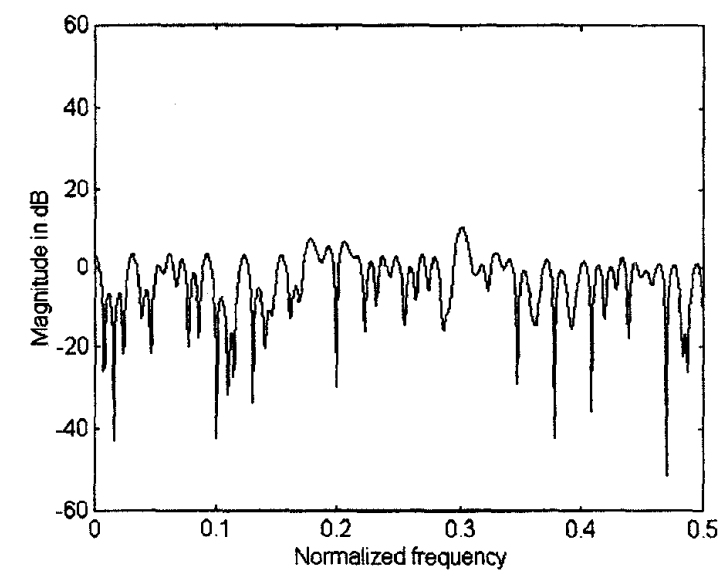

Fig. $2 a$

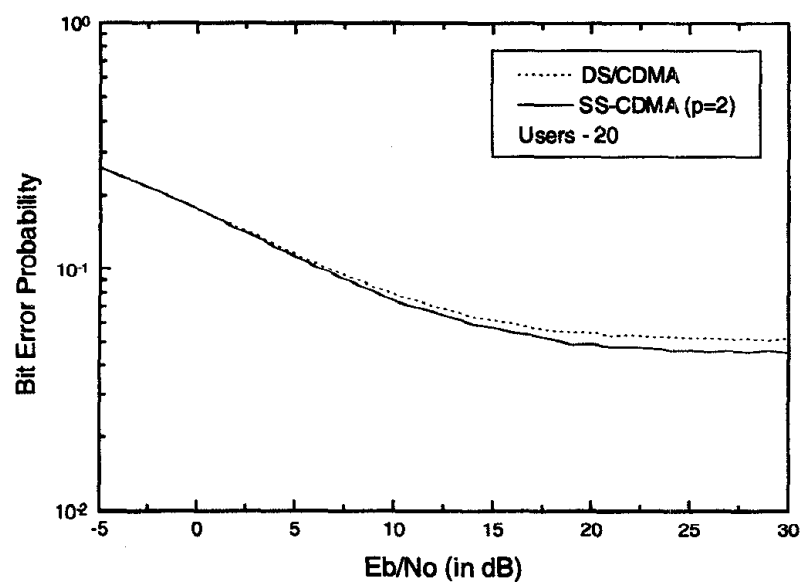

Fig .3 BER versus Eb/No (Uplink)

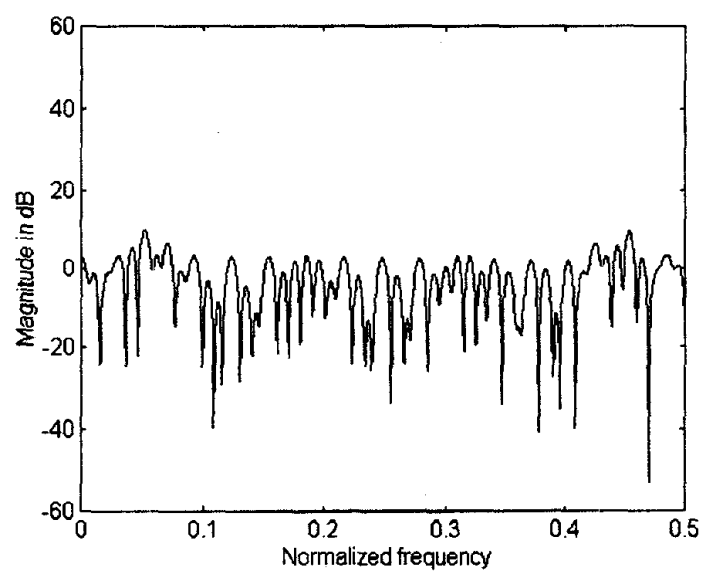

Fig. 2b

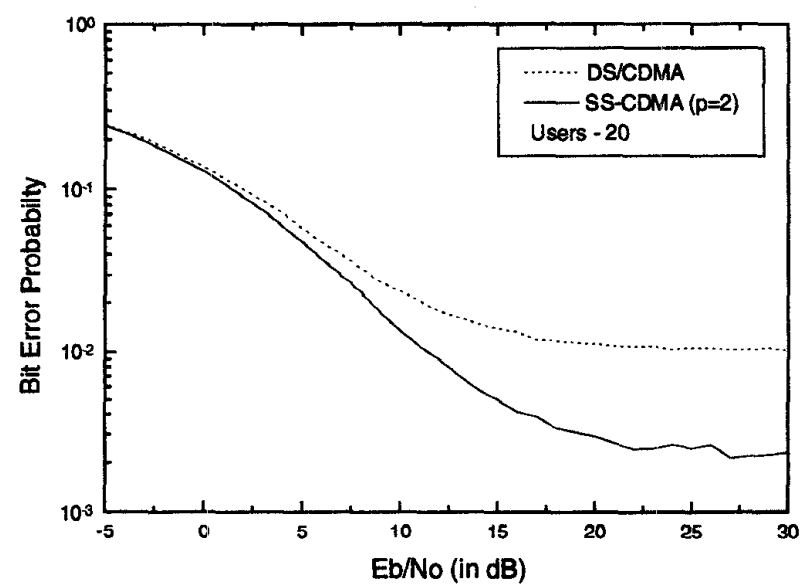

Fig. 4 BER versus Eb/No (down link) 